\title{
Adsorption Trajectories of Non-spherical Particles at Liquid Interfaces
}

\author{
S.O. Morgan ${ }^{1}$, J. Fox ${ }^{1,2}$, C. Lowe $^{1}$, A.M. Adawi ${ }^{1}$, J.-S.G. Bouillard ${ }^{1}$, G.J. Stasiuk ${ }^{3}$, T.S. Horozov ${ }^{4}$, D.M.A. Buzza ${ }^{1, *}$ \\ ${ }^{1}$ Department of Physics \& Mathematics, University of Hull, Hull HU6 7RX, U.K. \\ ${ }^{2}$ School of Physics 83 Astronomy, University of Leeds, Leeds LS2 9JT, U.K. \\ ${ }^{3}$ Imaging Chemistry 83 Biology, King's College London, Strand, London WC2R 2LS, U.K. \\ ${ }^{4}$ Department of Chemistry $\&$ Biochemistry, University of Hull, Hull HU6 7RX, U.K.
}

(Dated: March 29, 2021)

\begin{abstract}
The adsorption of colloidal particles at liquid interfaces is of great importance scientifically and industrially but the dynamics of the adsorption process is still poorly understood. In this paper, we use a Langevin model to study the adsorption dynamics of ellipsoidal colloids at a liquid interface. Interfacial deformations are included by coupling our Langevin dynamics to a finite element model while transient contact line pinning due to nanoscale defects on the particle surface is encoded into our model by renormalising particle friction coefficients and using dynamic contact angles relevant to the adsorption timescale. Our simple model reproduces the monotonic variation of particle orientation with time that is observed experimentally and is also able to quantitatively model the adsorption dynamics for some experimental ellipsoidal systems but not others. However, even for the latter case, our model accurately captures the adsorption trajectory (i.e., particle orientation vs. height) of the particles. Our study clarifies the subtle interplay between capillary, viscous and contact line forces in determining the wetting dynamics of micron-scale objects, allowing us to design more efficient assembly processes for complex particles at liquid interfaces.
\end{abstract}

\section{INTRODUCTION}

The adsorption of colloidal particles at liquid interfaces is of great importance for a wide range of applications ranging from emulsification [1], encapsulation [2], food and pharmaceuticals [3], nanostructured materials [4] and reconfigurable materials [5]. At a more fundamental level, it has also opened the door for the creation of exotic new states of matter such as liquid marbles [6], bijels [7, 8] and 'dry' water [9]. Since the pioneering work of Pieranski [10], the equilibrium behaviour of particles at liquid interfaces is well understood: the huge reduction in free energy upon particle adsorption (typically millions of $k T$ for micron-scale particles) provides a very strong driving force for adsorption, while the final equilibrium height of spherical particles relative to the liquid interface is determined by the equilibrium contact angle.

In contrast to the equilibrium situation, the dynamics of the adsorption of micron-scale colloids at liquid interfaces is much less understood. Intuitively, given the large driving force for adsorption, one would expect the adsorption process to be very fast. It was therefore a great surprise when Kaz et al. [11] found that, after initially breaching the liquid interface, spherical colloidal particles relax toward their equilibrium position at a rate that is orders of magnitude slower than what is predicted by models based on viscous dissipation [12-14]. However, they found that the logarithmically slow wetting dynamics could be explained if one accounts for the transient pinning of the three phase contact line at nanoscale defects on the colloid surface $[15,16]$. Subsequent studies have shown that the transient pinning and depinning of the contact line also dominates other dynamic processes of colloids at liquid interfaces, for example leading to anomalously slow in-plane Brownian diffusion [17].

The seminal work of Kaz et al. on spherical colloids has recently been extended to ellipsoidal particles by Wang et al. [18] and Coertjens et al. [19] and very slow adsorption dynamics were also found in this case. However, the adsorption kinetics of ellipsoids is more complicated because, in addition to particle height relative to the interface, there is the additional adsorption coordinate of particle orientation, and the final equilibrium state is where the particle is oriented horizontally along the interface [20]. Both experimental studies above found that particle orientation varies monotonically with time during particle relaxation towards equilibrium, in sharp contrast with current viscous-based models which predict a non-monotonic variation of particle orientation with time $[21,22]$. The qualitative difference between theory and experiment has led Wang et al. to propose contact line pinning not only controls the adsorption timescale, but also the adsorption trajectory (i.e., how particle orientation varies with particle height) of non-spherical particles [18].

However, it is important to note that the viscous model used by de Graaf et al. [21] did not take into account a number of important effects. Firstly, the ratio of the translational to rotational friction coefficients used in the model were physically unrealistic [21]. In addition, the model neglects the deformation of the liquid meniscus around the adsorbing particle though these deformations are almost certainly significant for particle adsorption in the low capillary number regime [20, 23-26]. It is therefore important to develop a theoretical model which incorporates these effects before we can accurately assess the role played by different driving forces (capillary, viscous, contact line pinning etc.) in determining the adsorption trajectory of non-spherical particles at liquid interfaces.

In principle, the particle-based simulations in ref.[22, 27] can meet some of these requirements, but it is 
not possible for current simulations to achieve the very long timescales required to access the low capillary and Reynolds number regimes that are relevant to the experiments. Alternatively, Wang et al [18] have developed a contact line hopping model $[11,15,16]$ that is able to accurately model their experimental data for the adsorption dynamics of ellipsoids. However, in order to make their calculations tractable, the authors have made the simplifying assumption that the liquid interface remains flat during particle adsorption. While this assumption appears to be reasonable for their experimental system (see ref.[18] and our discussion later), it is not physically realistic in general as discussed earlier [20, 23-26]. However, due to the complexity of the model, it is difficult to incorporate interfacial deformations within the framework of the contact line hopping model.

The aim of this paper is to address these challenges using a Langevin model for particle adsorption which incorporates interfacial deformation, the correct ratio for particle friction coefficients and contact line pinning. Specifically, interfacial deformations are included by coupling our Langevin dynamics to a finite element model [28-30] while contact line pinning is encoded into our model by renormalising particle friction coefficients [17] and using the dynamic contact angle relevant to the timescale of the adsorption process [11]. Surprisingly, this simple model is able to reproduce much of the reported experimental phenomenology. For example, our model reproduces the monotonic variation of particle orientation with time. Specifically, we were able to obtain this behaviour by accurately modelling interfacial deformation and particle friction coefficients without the need to explicitly invoke depinning dynamics of the contact line. Our model is also able to quantitatively model the adsorption dynamics (i.e., individual adsorption coordinates vs. time) observed by Coertjens et al. [19] but not by Wang et al. [18]. However, even in the latter case, we are able to accurately capture the adsorption trajectory (i.e., particle orientation vs. particle height) of the system.

Our model clarifies the different roles played by capillary, viscous and contact line forces in determining the wetting dynamics of micron-scale objects. Furthermore, since the final equilibrium state of anisotropic particles at the liquid interface is controlled by their adsorption trajectory rather than by the dynamics of the individual adsorption coordinate, our model can be used as a predictive tool for designing efficient assembly processes for complex particles at liquid interfaces.

\section{THEORETICAL MODEL}

We consider a prolate ellipsoidal particle adsorbing at a liquid interface, with long and short axis $a, b$ respectively and aspect ratio $m=a / b$ (see Figure 1 ). Following experiments, we refer to the top and bottom liquid phases as oil and water respectively. The configuration of the ellipsoid at any instant during adsorption is described

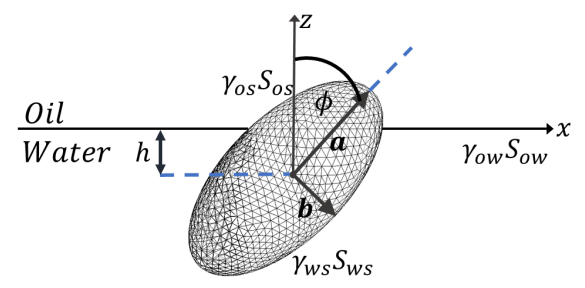

FIG. 1. Variables characterising the configuration of an ellipsoid adsorbing at a liquid interface.

by the generalised coordinates $h$ and $\phi$, where $h$ is the distance of the particle centre from the undeformed liquid interface and $\phi$ is the angle between the particle long axis and the flat interface normal. The particle height when it first contacts the liquid interface is given by $h_{c}(\phi)=b \sqrt{m^{2} \cos ^{2} \phi+\sin ^{2} \phi}[31]$ so that $h=+h_{c},-h_{c}$ corresponds to the particle touching the interface from the oil and water side respectively.

For micron-sized particles where gravity is negligible, the free energy of the system is given by [21, 28, 29]

$$
F=\gamma_{o w} S_{o w}+\gamma_{o s} S_{o s}+\gamma_{w s} S_{w s}
$$

where $\gamma_{o w}, \gamma_{o s}, \gamma_{w s}$ are the interfacial tensions and $S_{o w}$, $S_{o s}, S_{w s}$ are the areas of the oil/water, particle/oil and particle/water interfaces respectively. Using Young's equation $\gamma_{o w} \cos \theta_{w}=\gamma_{o s}-\gamma_{w s}$ where $\theta_{w}$ is the contact angle of the oil/water interface at the particle surface, noting that $S_{o s}=S_{p}-S_{w s}$ (where $S_{p}$ is the total area of the particle) and dropping irrelevant constant terms, we can simplify Eq. (1) to

$$
F=\gamma_{o w} S_{o w}+\gamma_{o w} \cos \theta_{w} S_{o s}
$$

Note that we neglect line tension in the above free energies as we have checked that it has a negligible effect on adsorption dynamics for experimentally measured values of the line tension for micron-sized ellipsoids [32]. This observation is also consistent with what other authors have found for micron-sized objects [27].

The interfacial areas and hence particle free energy depends sensitively on the boundary condition at the three phase contact line. We consider two limiting cases, firstly where the liquid interface remains flat, secondly where it is deformed due to the constant contact angle requirement $[28,29]$. The free energy in both cases is calculated using the finite element package Surface Evolver [28-30]. Specifically, the value of $F(h, \phi)$ is calculated on a $101 \times 37$ non-equidistant grid for $h \in\left[-h_{c}, h_{c}\right]$ and $\phi \in[0, \pi / 2]$; the data on this grid are then interpolated with a third order interpolation scheme to yield the full free energy landscape.

The adsorption trajectory is found by solving the Langevin equation for the particle at the liquid interface. In the low Reynolds number regime where inertial forces are negligible, this is given by the coupled differential 
equations

$$
\begin{aligned}
\lambda \frac{d h}{d t} & =-\frac{\partial}{\partial h} F(h, \phi) \\
\mu \frac{d \phi}{d t} & =-\frac{\partial}{\partial \phi} F(h, \phi)
\end{aligned}
$$

where $\lambda, \mu$ are the translational and rotational friction coefficient of the ellipsoid respectively. The left and right hand side of the above equations are the frictional and capillary forces respectively associated with translational (Eq. (3)) and rotational (Eq. (4)) motion. Note that since we are considering particles at liquid interfaces, $\lambda$ and $\mu$ will include contributions from both viscous forces due to the bulk phases as well as contact line forces from the interface [17]. Note also that we have neglected random forces in the above equations since they are subdominant compared to capillary forces at a liquid interface.

In the general case, the friction coefficients $\lambda$ and $\mu$ are functions of $h$ and $\phi$ due to the assymmetry in the viscosity of the bulk phases and the anisotropy of the particle. However, as we shall see later, the frictional forces in the experimental systems are dominated by contact line forces rather than viscous forces. To a first approximation, we can therefore assume that both $\lambda$ and $\mu$ are independent of $h$, i.e., the degree to which the particle is immersed in each of the two phases. We also note that the translational friction coefficient along the long and short axis of the ellipsoid differ by at most a factor of 2 in a viscous-based model [21,33]. To a first approximation, we will therefore also assume that $\lambda$ and $\phi$ are independent of $\phi$. Note that we have included the $\phi$ dependence of $\lambda$ explicitly in our Langevin model [33] and found that this leads to adsorption trajectories which are essentially the same as what we obtain for constant $\lambda$.

Assuming $\lambda, \mu$ are independent of $h, \phi$ therefore, we can rescale Eqs. (3),(4) to

$$
\begin{aligned}
& \frac{d h^{*}}{d t^{*}}=-\frac{\partial}{\partial h^{*}} F^{*}\left(h^{*}, \phi^{*}\right) \\
& \frac{d \phi^{*}}{d t^{*}}=-\frac{\partial}{\partial \phi^{*}} F^{*}\left(h^{*}, \phi^{*}\right),
\end{aligned}
$$

or more compactly to

$$
d \vec{\eta}\left(t^{*}\right) / d t^{*}=-\vec{\nabla} F^{*}\left(h^{*}, \phi^{*}\right) .
$$

In the above equations, $h^{*} \equiv h / \alpha, t^{*}=t / \beta, \phi^{*}=$ $\phi / \pi, F^{*}=F / \gamma_{o w} b^{2}$ are scaled variables, $\alpha, \beta$ are scale factors that will be discussed in a moment, $\vec{\eta}\left(t^{*}\right)=$ $\left(h^{*}\left(t^{*}\right), \phi^{*}\left(t^{*}\right)\right)$ is the dynamical state vector of the particle at any given moment in time and $\vec{\nabla}=\left(\frac{\partial}{\partial h^{*}}, \frac{\partial}{\partial \phi^{*}}\right)$ is the grad operator in $\left(h^{*}, \phi^{*}\right)$ coordinate space. Eq. (7) tells us that the adsorption trajectories for the particle are remarkably simple in our simplified Langevin dynamics, i.e., they follow the path of steepest descent in the free energy landscape $F^{*}\left(h^{*}, \phi^{*}\right)[21]$.

The dynamic scale factors $\alpha$ and $\beta$ depend on the friction coefficient ratio $\mu / \lambda$. In ref.[21], de Graaf et al. chose $\alpha=\sqrt{a^{2}+2 b^{2}}, \beta=\frac{a^{2}+2 b^{2}}{\gamma_{o w} b^{2}} \lambda$ which corresponds to $\frac{\mu}{\lambda}=\frac{a^{2}+2 b^{2}}{\pi^{2}}$; we call this choice of scale factors Scaling 1 . However, these authors point out that this choice is unphysical for a viscous-based model because it does not yield the sphere value $\frac{\mu}{\lambda}=\frac{4 b^{2}}{3}$ for $m=1$ [21]. To overcome this problem, we also consider the scale factors $\alpha=\frac{2 \pi}{3} \sqrt{a^{2}+2 b^{2}}$ and $\beta=\frac{4 \pi\left(a^{2}+2 b^{2}\right)}{9 \gamma_{o w} b^{2}} \lambda$ which corresponds to $\frac{\mu}{\lambda}=\frac{4\left(a^{2}+2 b^{2}\right)}{9}$; we call this choice of scale factors Scaling 2 .

\section{RESULTS AND DISCUSSIONS}

To study the impact of different contact line boundary conditions and dynamic scaling on adsorption kinetics, in Figure 2a-c we show the adsorption trajectories calculated from our Langevin model for the simple case of neutrally wetting ellipsoids $\left(\theta_{w}=90^{\circ}\right)$ with $m=2$ for a flat liquid interface and Scaling 1 (Figure 2a), a flat interface and Scaling 2 (Figure 2b) and a deformed interface and Scaling 2 (Figure 2c). Regardless of the initial angle of the particle, we see that all adsorption trajectories that start from particle contact with the liquid interface (left/right red curve corresponds to contact from the water/oil side) end at the equilibrium state $(h=0, \phi=\pi / 2)$ where the ellipsoid lies flat along the interface. However, assuming a flat liquid interface leads to trajectories that are non-monotonic in $\phi$ (Figures $2 \mathrm{a}$ and $2 \mathrm{~b}$ ) while assuming a deformed liquid interface leads to trajectories are monotonic in $\phi$ (Figure 2c).

This qualitative change in the variation of $\phi$ is due to the fact that different contact line boundary conditions lead to very different free energy landscapes as shown in Figure 2d (flat interface) and Figure 2e (deformed interface). Recall that we are in the low capillary number regime where the adsorption is essentially a quasi-static process, i.e., particle adsorption is slow enough for the liquid interface to be in equilibrium with the configuration of the ellipsoid at each stage of the adsorption process. This means that when the liquid interface can deform, immediately after the particle breaches the liquid interface at $h= \pm h_{c}(\phi)$, the ellipsoid is attached to the liquid interface (effectively instantaneously on particle adsorption timescales) in order to satisfy the constant contact angle condition (Figure 2e left). In this case, the free energy of the system at $h= \pm h_{c}(\phi)$ strongly depends on $\phi$, for example the horizontal state of the ellipsoid ( $\phi=\pi / 2)$ has lower free energy compared to the vertical state $(\phi=0)$ since the former excludes more liquid interface compared to the latter. In contrast, when the liquid interface is flat, the ellipsoid is essentially detached from the liquid interface for $h= \pm h_{c}(\phi)$ (Figure 2d left) and the free energy of the system at $h= \pm h_{c}(\phi)$ is independent of $\phi$. The flat interface assumption thus effectively raises up the corners of the landscape at $\phi=\pi / 2$, causing the steepest descent paths to initially decrease in $\phi$. The non-monotonic trajectories in Figure 2a,b are there- 
(a)
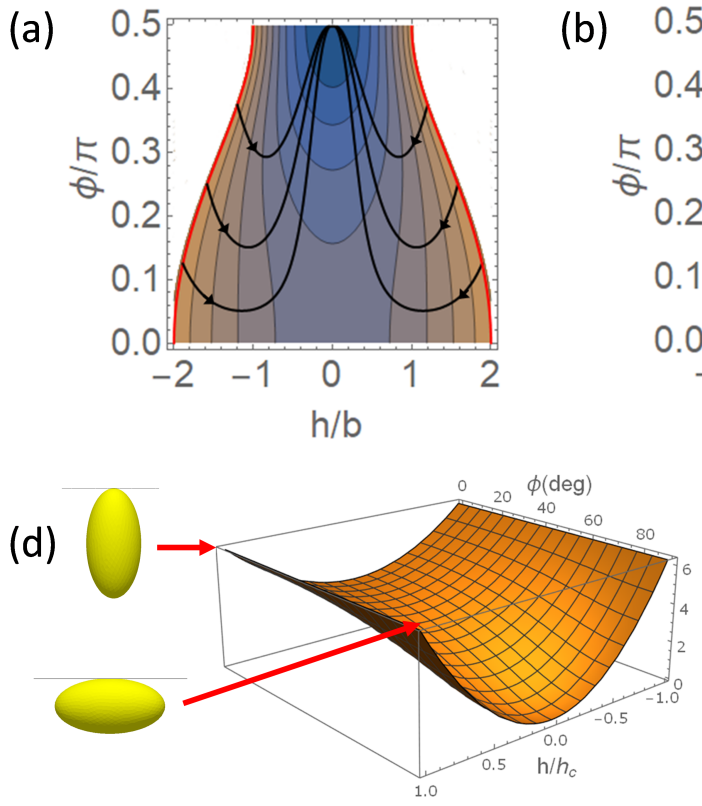

(b)

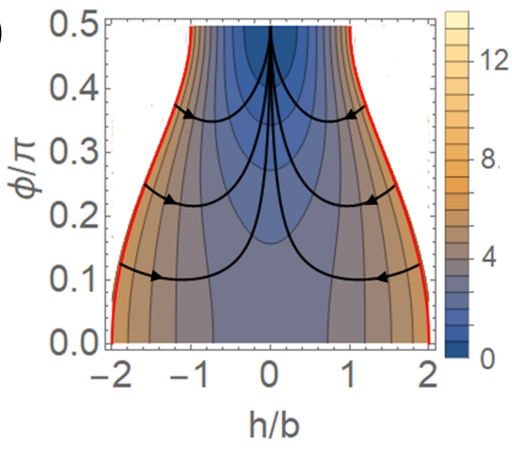

(c)

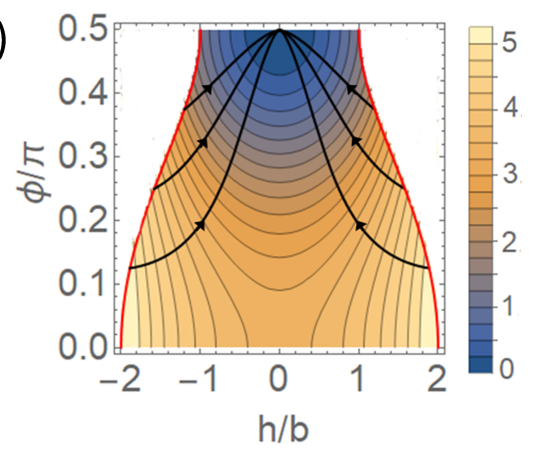

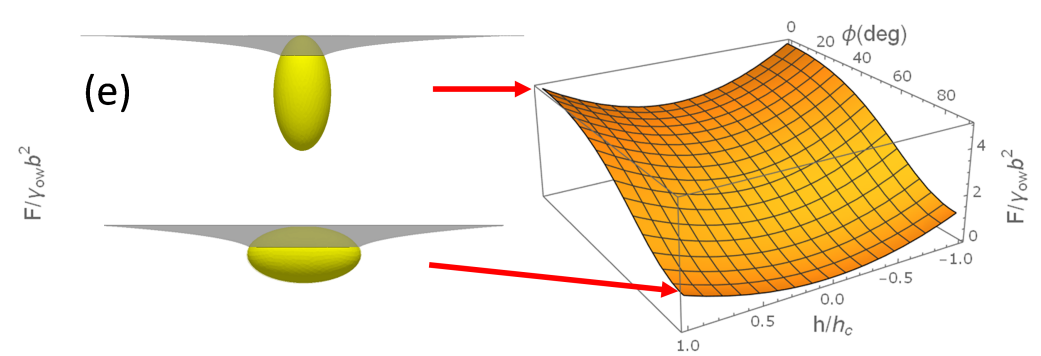

FIG. 2. (a)-(c) Adsorption trajectories in the $(h, \phi)$ plane (black lines) for ellipsoids with contact angle $\theta_{w}=90^{\circ}$, aspect ratio $m=2$ for: (a) flat interface and Scaling 1; (b) flat interface and Scaling 2; (c) deformed interface and Scaling 2 . The trajectories are superposed on contour plots of free energy landscapes and the red curves bounding the landscape correspond to particles touching the interface from the oil side $\left(h=+h_{c}(\phi)\right)$ or water side $\left(h=-h_{c}(\phi)\right)$. For illustrative purposes, we show trajectories starting from either the oil or water side with initial particle angles $\phi_{0}=22.5^{\circ}, 45^{\circ}, 67.5^{\circ} ;(\mathrm{d})-(\mathrm{e})$ Free energy landscape represented as a three-dimensional plot of free energy vs. $h / h_{c}$ and $\phi$ and system configuration at $h=-h_{c}$ for ellipsoids in the vertical or horizontal orientation for: (d) flat interface case; (e) deformed interface case.

fore an artefact of the flat interface assumption, and such trajectories are either strongly suppressed or disappear altogether when we relax this assumption.

We next compare our theoretical model with the experiments of Coertjens et al. for fluorescent polystyrene ellipsoids with $m \approx 4$ at an oil/water interface [19]. The appropriate contact angle $\theta_{w}$ we should use for this system is a delicate question since the contact angle evolves slowly with time due to physical aging of the contact line [32]. To account for this effect, we use as our effective contact angle the dynamic (rather than equilibrium) contact angle that is relevant to the timescale of the adsorption process. Specifically, since adsorption occurs on the $\sim 0.3 \mathrm{~s}$ timescale in this case, and the dependence of $\theta_{w}$ on particle stretching is weak [32], we use the contact angle measured for equivalent fluorescent polystyrene spheres on a similar timescale which is $\theta_{w} \approx 90^{\circ}[32]$. Note that strictly speaking, one should also account for differences in advancing and receding angles around the contact line during particle adsorption [18]. However, as we are seeking a minimal model to capture the essential features of the experimental system, we have neglected this difference to a first approximation.

Note that due to limitations of their high speed confocal microscopy method, Coertjens et al. were only able to measure $\phi$ as a function of time [19]. In Figure 3, we compare all three models discussed above with a linear$\log$ plot of the $\phi$ vs. $t$ data of Coertjens et al. for an initial particle angle of $\phi_{0} \approx 30^{\circ}$. We use $\beta$ as our fitting parameter to fit the drop in the data away from $\phi=90^{\circ}$. We see that quantitative agreement with the experimental data is obtained using the deformed interface and Scaling 2 model (black solid curve) but not for the flat interface models, suggesting that the interface is deformed rather than flat during particle adsorption. Note that in Figure 3, the experimental relaxation data for $\phi$ is clearly non-logarithmic.

Using the fitted value of $\beta$ for the black curve $(\beta=$ $16 \mathrm{~s})$ and assuming $\gamma_{o w}=50 \mathrm{mN} \cdot \mathrm{m}^{-1}$ yields $\lambda=$ $10^{-2} \mathrm{~kg} \cdot \mathrm{s}^{-1}$. It is instructive to compare this value for $\lambda$ with that due to the bulk fluid viscosity. For prolate ellipsoids with $m>2$, the rotational friction coefficient due to the viscosity of the surrounding medium $\eta$ can be approximated by [33, 34]

$$
\mu=\frac{16 \pi \eta a^{3}}{3[2 \ln (2 m)-1]} .
$$

Combining the above equation with the ratio $\frac{\mu}{\lambda}=$ $\frac{4\left(a^{2}+2 b^{2}\right)}{9}$ assumed in Scaling 2, the translational friction coefficient is given by

$$
\lambda=\frac{12 \pi \eta a^{3}}{\left(a^{2}+2 b^{2}\right)[2 \ln (2 m)-1]} .
$$

For the experimental system of Coertjens et al. [19], $\eta=20 \mathrm{mPa} \cdot \mathrm{s}, a=1200 \mathrm{~nm}, b=300 \mathrm{~nm}$ and $m=4$. Inserting these values into Eq. (9), we obtain $\lambda=2.5 \times$ 
$10^{-7} \mathrm{~kg} \cdot \mathrm{s}^{-1}$ which is more than four orders of magnitude smaller than the value obtained from fitting the experiments in Figure 3. This huge discrepancy between the viscous model and experiment suggests that the adsorption dynamics in these experiments is not controlled by viscous forces but (as we shall see in a moment) by contact line forces. The good fit between our Langevin model and experiment suggests that for this system, we can quantitatively model the effect of contact line pinning by renormalising the friction coefficient of the particle, similar to what was found by Boniello et al. for the in-plane diffusion coefficient of particles at a liquid interface [17]. Note that the renormalisation of friction coefficients due to contact line pinning is not merely a phenomenological fitting exercise. Instead, a microscopic basis for this procedure has been provided by Boniello et al. who explicitly showed that taking into account the thermally activated fluctuations of the contact line via the fluctuationdissipation theorem leads to significant enhancements of particle friction coefficients [17].

Adapting their approach to our problem, the contribution from contact line fluctuations to the translational friction coefficient is given by the fluctuation-dissipation theorem as [35]

$$
\lambda=\frac{1}{2 k_{B} T}\left\langle f(0)^{2}\right\rangle \tau_{c}
$$

where $k_{B} T$ is the thermal energy, $f(0)$ is the instantaneous surface tension force exerted by the liquid interface on a triple-line segment of length $\ell_{D}$ between neighbouring nanoscale surface defects and $\tau_{c}$ is the correlation time of the fluctuations. The random nature of the fluctuations allows us to write the mean squared force as

$$
\left\langle f(0)^{2}\right\rangle \approx n\left(\gamma_{o w} \ell_{D}\right)^{2}
$$

where $n=2 \pi\left[\left(a^{2}+2 b^{2}\right) / 3\right]^{1 / 2} / \ell_{D}$ is the number of uncorrelated triple-line segments around the ellipsoid. On the other hand, the correlation time due to the thermally activated jumps of the contact line has the Arrhenius-like form [36]

$$
\tau_{c} \approx \frac{\eta V_{m}}{k_{B} T} \exp \left[\frac{\ell_{D}^{2} \gamma_{o w}\left(1+\cos \theta_{w}\right)}{k_{B} T}\right]
$$

where $V_{m} \approx 3 \times 10^{-29} \mathrm{~m}^{3}$ is the molecular volume of water.

Substituting Eqs. (11),(12) into Eq. (10), we obtain an expression for the friction coefficient $\lambda$ involving only one fitting parameter, namely the distance between surface nanoscale defects $\ell_{D}$. Using the value $\lambda=10^{-2} \mathrm{~kg} \cdot \mathrm{s}^{-1}$ obtained from fitting the experimental data in Figure 3 and the experimental parameters for ref.[19] discussed above, we obtain $\ell_{D} \approx 0.9 \mathrm{~nm}$. This nanometric value for $\ell_{D}$ is physically reasonable and provides support that contact line pinning is indeed what gives rise to the significantly enhanced value for the friction coefficient.

We next compare our theoretical model with the experiments of Wang et al. [18] for polystyrene ellipsoids

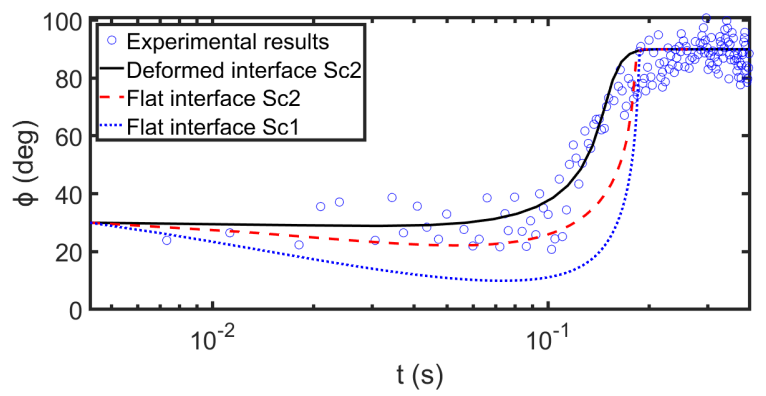

FIG. 3. Linear-log plot of $\phi$ vs. $t$ comparing the three theoretical models discussed in the main text for ellipsoids with $m=4, \theta_{w}=90^{\circ}$ with the experimental data of Coertjens et al. [19].

with $m \approx 2.6$ [37] at an oil/water interface. The adsorption process for this system is much faster, occurring on the $\sim 0.03 \mathrm{~s}$ timescale. Once again, for $\theta_{w}$ we use the dynamic contact angle measured for an equivalent sphere on this timescale which is $\theta_{w} \approx 45^{\circ}$ [11]. The digital holography technique used by Wang et al. is capable of measuring both $\phi$ and $h$ as a function of time, allowing us to compare theory and experiment for both the dynamics of the individual coordinates (i.e., $\phi$ or $h$ vs. $t$ ) and the adsorption trajectory (i.e., $\phi$ vs. $h$ ); the latter representation of the data is particularly useful as it allows us to perform a parameter-free comparison between theory and experiment.

In Figure 4a,b, we compare all three models discussed above with linear-log plots of the experimental data for the average value of $\phi$ vs. $t$ (Figure $4 \mathrm{a}$ ) and $h$ vs. $t$ (Figure 4b). We note that, in contrast to Figure 3, the experimental dynamics of the individual adsorption coordinates are logarithmic. We also note that none of the three theoretical models can reproduce the experimental data in Figure 4, even if we renormalise the friction coefficients to account for contact line pinning. The discrepancy between theory and experiment is perhaps not surprising since, as pointed out in refs.[11, 18], it is not possible for a Langevin model where dissipative forces are parameterised by a handful of friction coefficients to generate the hierarchy of timescales required for logarithmic dynamics. However, the discrepancy between theory and experiment seen in Figure 4 also highlights the fact that the contact line dynamics in the experiments of Wang et al. [18] is qualitatively different from that of Coertjens et al. [19] (Figure 3). We will discuss possible reasons for this difference at the end of this section.

However, while the theoretical models are not able to capture the dynamics of the individual adsorption coordinates, in Figure 4c we see that they are able to capture adsorption trajectory (i.e., $\phi$ vs. $h$ plot) of the experimental system surprisingly well. In particular, all three models are able to reproduce the essentially linear relationship between $\phi$ and $h$ found experimentally with no fitting parameters. It is also interesting that apart from 
(a)

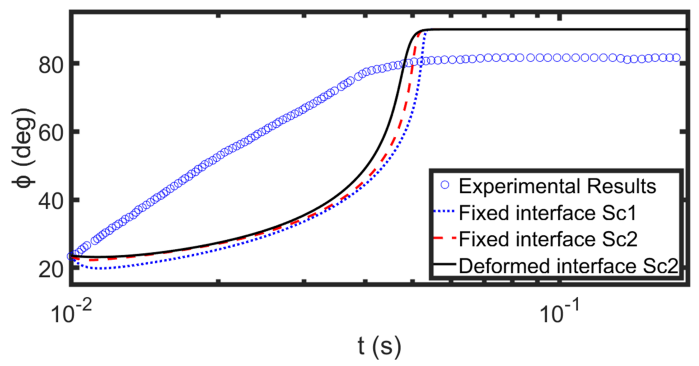

(b)

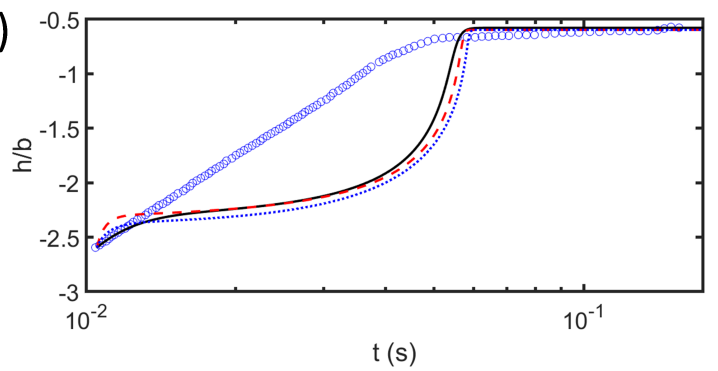

(c)

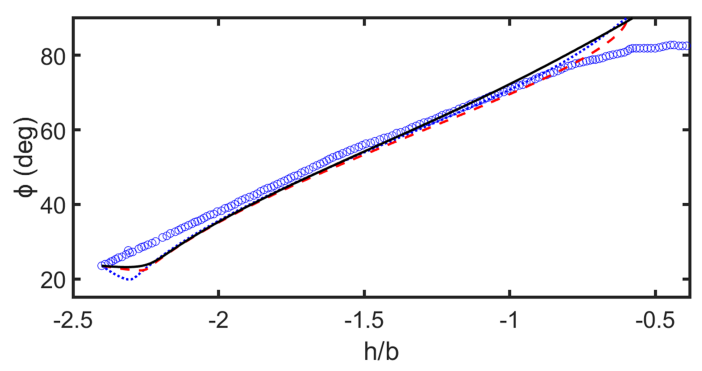

FIG. 4. Comparison of the three theoretical models discussed in the main text for ellipsoids with $m=2.6, \theta_{w}=45^{\circ}$ with the experimental data of Wang et al. [18] for (a) linear-log plot of $\phi$ vs. $t$; (b) linear-log plot of $h$ vs. $t$; (c) $\phi$ vs. $h$.

small discrepancies in the early stage dynamics, all three models predicts very similar adsorption trajectories.

We believe that the similarity between the flat and deformed interface models (with Scaling 2) in Figure 4c is due to the fact that the interfacial deformation during particle adsorption is small when we are far from the neutrally wetting regime (recall that the effective contact angle relevant to the experiments in ref.[18] is $\theta_{w} \approx 45^{\circ}$ ). This is illustrated in Figure 5 where we show the deformed liquid interface around an ellipsoid with $m=2.6$, $\phi=\pi / 2, h=-h_{c}$ for contact angle $\theta_{w}=45^{\circ}$ (Figure $5 \mathrm{a}$ ) and $\theta_{w}=90^{\circ}$ (Figure $5 \mathrm{~b}$ ). We see that deformation of the liquid interface for $\theta_{w}=45^{\circ}$ is much smaller compared to $\theta_{w}=90^{\circ}$ (average height of contact line is $-0.289 b$ and $-0.734 b$ in Figure $5 \mathrm{a}$,b respectively). The small interfacial deformation far from the neutrally wetting regime may also explain why the flat interface approximation works so well in modelling the experimental data of Wang et al. [18]. However, we emphasize that the flat interface approximation is not accurate in general. Specifically, close to the neutrally wetting con- (a)

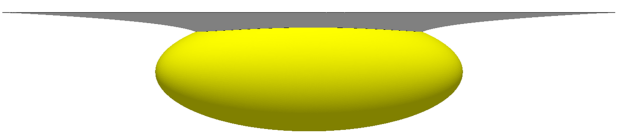

(b)

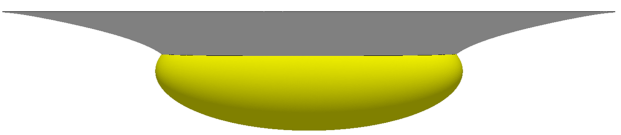

FIG. 5. Interfacial deformation calculated from Surface Evolver for an ellipsoid with $m=2.6, \phi=\pi / 2, h=-h_{c}$ for contact angle: (a) $\theta_{w}=45^{\circ}$ and (b) $\theta_{w}=90^{\circ}$.

dition, significant discrepancies emerge between the flat and deformed interface models (see Figure 3).

The similarity between the Scaling 1 and Scaling 2 models (with flat interface) in Figure 4c is most likely due to the fact that far from the neutrally wetting regime, all particle adsorption flow lines converge onto a 'dynamical attractor' after the initial stages of the adsorption [21]. This is illustrated in Figures 6a-c where we clearly see the emergence of an attractor in the adsorption flow lines for all three models. The attractor is formally defined as the locus of points in the free energy landscape $F^{*}\left(h^{*}, \phi^{*}\right)$ where one of the eigenvectors of the Hessian matrix $\vec{\nabla} \vec{\nabla}^{T} F^{*}$ (the one with positive eigenvalue or principal curvature) is parallel to the gradient of the free energy [21] and the resultant attractor is in general sensitive to the dynamic scaling assumed. However, far from the neutrally wetting regime, the free energy landscape develops a narrow valley (see Figures 6a-c), and since attractors are constrained to be in the vicinity of this valley, the attractors effectively become insensitive to the dynamic scaling used. This point is illustrated in Figure $6 \mathrm{~d}$ where we see that the attractors from all three models essentially the same. This explains why apart from small discrepancies in the early stage dynamics, the Scaling 1 and Scaling 2 models with flat interface (and indeed Scaling 2 model with deformed interface) predict very similar adsorption trajectories in Figure 4c.

The fact that far from the neutrally wetting regime, dynamic attractors are essentially determined by the geometry of the free energy landscape rather than the dynamic scaling model may also explain why in Figure 4c, all three theoretical models are able to capture the main features of the experimental adsorption trajectory even though they cannot capture the dynamics of the individual adsorption coordinates. This point is evidenced in Figure $6 \mathrm{~d}$ where we see that, after the initial stages of the adsorption, the experimental adsorption trajectory (data points) largely coincides with the dynamic attractors of all three models (lines).

Another reason why our theoretical models are able to capture the adsorption trajectory in Figure 4c even 

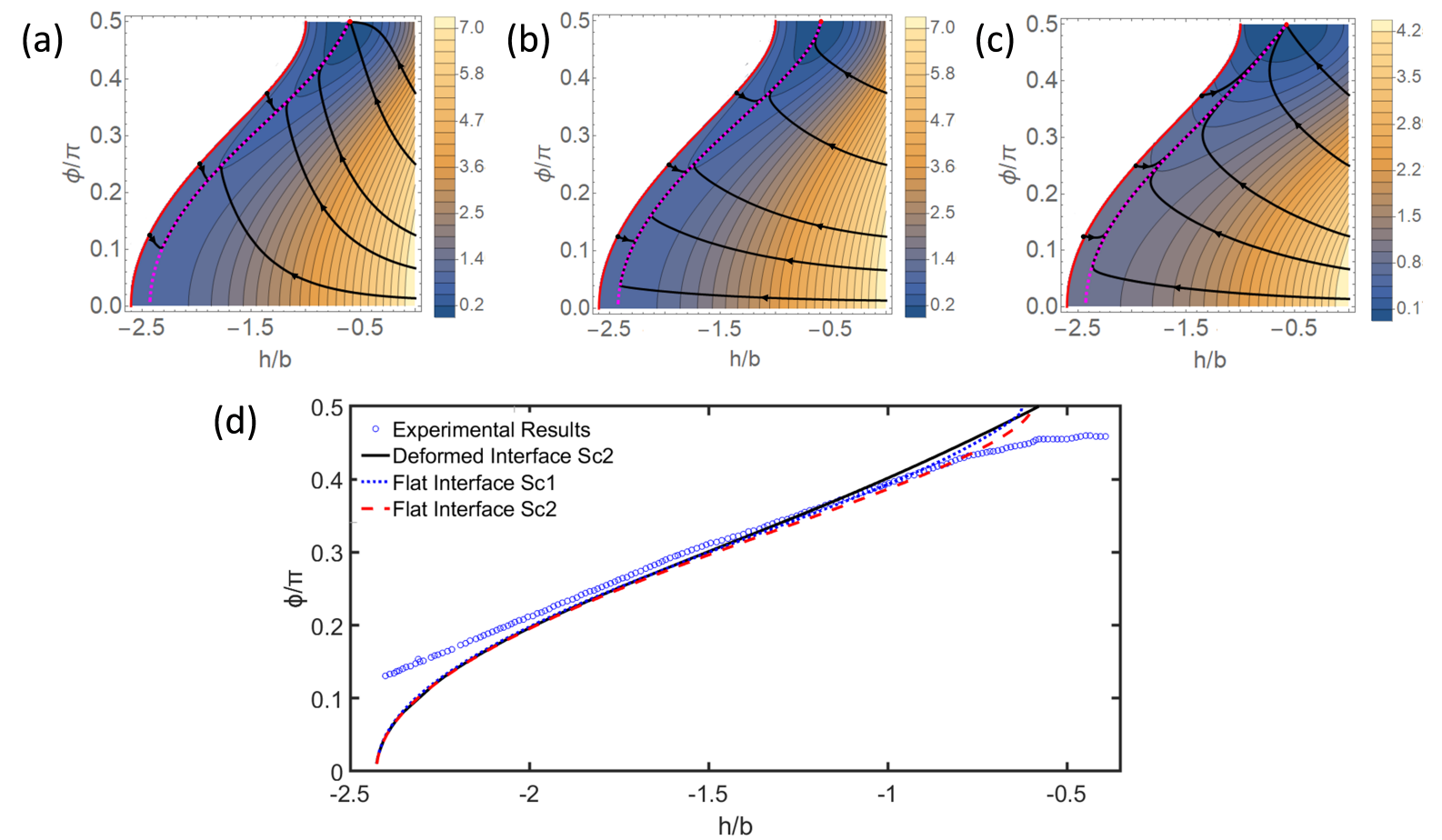

FIG. 6. (a)-(c) Adsorption trajectories in the $(h, \phi)$ plane, $h<0$ (black lines), for ellipsoids with contact angle $\theta_{w}=45^{\circ}$, aspect ratio $m=2.6$ for: (a) flat interface and Scaling 1; (b) flat interface and Scaling 2; (c) deformed interface and Scaling 2. The trajectories are superposed on contour plots of free energy landscapes and the red curve bounding the landscape corresponds to particles touching the interface from the water side. Note that in (a)-(c), the adsorption trajectories converge onto dynamical attractors which roughly lie along the valley of the free energy landscape; (d) Comparison of dynamical attractors from (a)-(c) with the experimental adsorption trajectories for polystyrene ellipsoids from Wang et al. [18].

though they cannot capture the time dependence of the individual adsorption coordinates may be because the coupling between $\phi$ and $h$ in our Langevin model comes from the capillary forces alone, and these forces depend only on particle configuration and are independent of the frictional forces (see Eqs. (3),(4)). This means that these models may still be able to capture the coupling between $\phi$ and $h$ accurately even if they do not correctly capture the frictional forces resisting particle adsorption.

Finally, we note that the experimental trajectory deviates from the theoretical models in the late stages of the adsorption in Figures $4 \mathrm{c}$ and $6 \mathrm{~d}$. This deviation is most likely due to the fact that when the experimental system is close to the equilibrium tilt angle of $\phi=90^{\circ}$, the capillary force driving particle adsorption becomes too small to overcome contact line pinning so that the ellipsoid becomes kinetically arrested and therefore cannot reach the equilibrium tilt angle [11].

Before leaving this section, we return to the interesting question about why the contact line dynamics seen in the experiments of Wang et al. [18] are so different from that of Coertjens et al. [19]. To frame this discussion in a broader context, it is interesting that contact line dynamics can be modelled by renormalising friction coefficients in some systems, e.g., refs.[17, 19], but not others, e.g., refs. $[11,18]$. We speculate that this difference could be due to large differences in the nanoscale surface defect density between the two sets of systems, which in turn lead to very different contact line dynamics. For example, for the polystyrene spheres at the air/water interface studied in ref.[17], the area per defect was determined to be $A=\ell_{D} \approx 0.2 \mathrm{~nm}^{2}$, while for the polystyrene spheres at the oil/water interface studied in ref.[11], the area per defect was determined to be in the range $A \approx 5-30 \mathrm{~nm}^{2}$. Interestingly, for the polystryrene ellipsoids studied in ref.[19], from the data in Figure 3 we determined a rather small area per defect of $A=\ell_{D} \approx 0.8 \mathrm{~nm}^{2}$, consistent with our speculation above.

In order to test this hypothesis, in the Appendix we use the contact line hopping model of Kaz et al. [11] to calculate the contact line dynamics (i.e., particle height vs. time) for the two spherical particle systems discussed above. In Figure 7a, we show the linear-log plot of the normalised height of the sphere $\bar{z}$ vs. the normalised time $\bar{t}$ for sulphate terminated polystyrene spheres at an oil/water interface studied by Kaz et al. [11] which have $A \approx 5 \mathrm{~nm}^{2}$ (see Appendix for further details). We see that the relatively large value of $A$ in this case leads to an essentially logarithmic time dependence for particle height. On the other hand, in Figure $7 \mathrm{~b}$, we show the linear$\log$ plot of $\bar{z}$ vs. $\bar{t}$ for sulphate or amidine terminated polystyrene spheres at an air/water interface studied by 

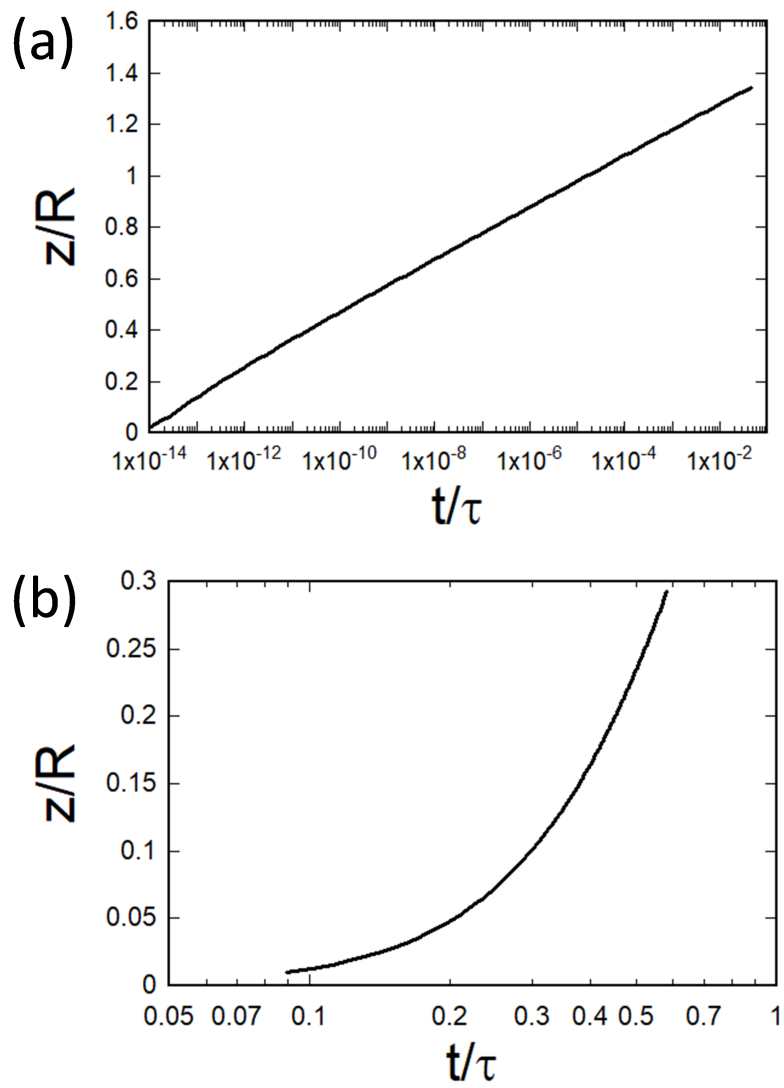

FIG. 7. Linear-log plot of normalised height $\bar{z}$ vs. normalised time $\bar{t}$ for micro-spheres adsorbing at a liquid interface calculated from contact-line hopping model for experimental parameters from systems studied by (a) Kaz et al. [11]; (b) Boniello et al. [17].

Boniello et al. [17] which have $A \approx 0.18 \mathrm{~nm}^{2}$ (again see Appendix for further details). We see that the relatively small value of $A$ in this case leads to a non-logarithmic time dependence for particle height.

Note that contact line pinning may arise from nanoscale surface features due to chemical [11], topological [32] or charge [38, 39] heterogeneities. This means that small differences in surface chemistry or sample preparation protocols may lead to large differences in contact line dynamics, even between systems which are nominally similar. Regardless of the exact microscopic origin for the different contact line dynamics, what seems clear from comparing the different experimental data on interfacial colloid dynamics is that while contact line pinning always leads to slower wetting dynamics, the logarithmic time dependence may not be universal but may be system dependent. This may explain why contact line dynamics can be modelled by renormalising friction coefficients in some systems but not others.

\section{CONCLUSIONS}

In summary, we have developed a simple Langevin model which accurately captures the deformation of the liquid meniscus and uses the correct ratio for the translational and rotational particle friction coefficients. The effect of contact line pinning is incorporated into the model by renormalising particle friction coefficients and using the appropriate dynamic contact angle. Using this simple model, we were able to reproduce the monotonic variation of particle orientation with time that is observed experimentally. Specifically, we were able to obtain this behaviour by accurately modelling interfacial deformation and particle friction coefficients without the need to explicitly invoke depinning dynamics of the contact line. We were also able to quantitatively model the adsorption dynamics of the individual adsorption coordinates (i.e., adsorption coordinate vs. time) for some experimental ellipsoidal systems but not others. However, even for the latter case, our model was able to accurately capture the adsorption trajectory (i.e., particle orientation vs. height) of the particles.

Our model clarifies the different roles played by capillary, viscous and contact line forces in determining the wetting dynamics of micron-scale objects. Note that since the final equilibrium state of the anisotropic particle is controlled by its adsorption trajectory rather than by the adsorption dynamics of the individual coordinates, our theoretical model can be used as a predictive tool for designing and controlling the assembly of complex particles at liquid interfaces and for future work we plan to use it to study the adsorption of particles with other non-spherical geometries.

\section{ACKNOWLEDGMENTS}

This work has received funding from the University of Hull PhD Scholarship Scheme (SOM, CL), Excel Communications (JF) and the European Union's Horizon 2020 research and innovation programme under grant agreement No 861950, project POSEIDON. We thank Vinny Manoharan, Anna Wang, Lucio Isa, Jens Harting and Ken Brakke for fruitful discussions. We also acknowledge the Viper High Performance Computing facility of the University of Hull and its support team.

\section{Appendix A: Adsorption dynamics from contact line hopping model}

In this appendix, we calculate the adsorption dynamics of a sphere at a liquid interface using the contact line hopping model of Kaz et al. [11]. The configuration of a sphere of radius $R$ at any instant during adsorption can be described by the height of the particle apex above the liquid interface $z$ (see Figure 8). Assuming the liquid interface remains flat during particle adsorption, $z$ is 


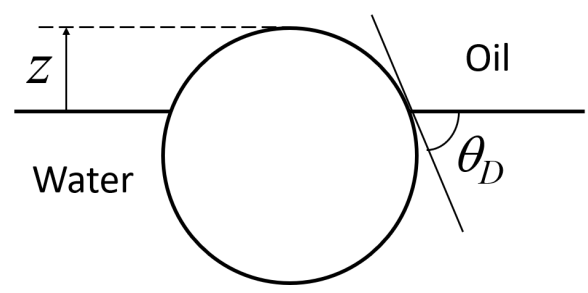

FIG. 8. Variables used in the model of Kaz et al. [11] to characterise the configuration of a sphere adsorbing at a liquid interface.

related to the dynamic contact angle $\theta_{D}$ by

$$
z=R\left(1-\cos \theta_{D}\right) .
$$

Using the contact line hopping model of Blake et al. [15], Kaz et al. derived the following equation of motion for $z$

$$
R \dot{z}=\sqrt{z(2 R-z)} V_{0} e^{-U / k T+\gamma A\left(\cos \theta_{D}-\cos \theta_{E}\right) / 2 k T}
$$

where $\dot{z}$ is the $z$ velocity of the particle, $k T$ is the thermal energy, $V_{0}$ is the molecular hopping speed, $U$ is the energy barrier to molecular hopping at the contact line due to nanoscale surface defects on the colloid, $A$ is the area per surface defect, $\gamma$ is the surface tension of the liquid interface and $\theta_{E}$ is the equilibrium contact angle. Substituting Eq. (A1) into Eq. (A2), we can replace $\theta_{D}$ with $z$ to get

$$
R \dot{z}=\sqrt{z(2 R-z)} V_{0} \exp \left[-\frac{U}{k T}+\frac{\gamma A}{2 k T} \frac{\left(z_{E}-z\right)}{R}\right]
$$

where $z_{E}=R\left(1-\cos \theta_{E}\right)$. Finally, using scaled variables $\bar{z}=z / R, \bar{t}=t / \tau$ where $\tau^{-1}=\frac{V_{0}}{R} \exp \left(-\frac{U}{k T}\right)$, we can write Eq. (A3) more compactly as

$$
\frac{d \bar{z}}{d \bar{t}}=\sqrt{\bar{z}(2-\bar{z})} \exp \left[a\left(\bar{z}_{E}-\bar{z}\right)\right]
$$

where $a=\frac{\gamma A}{2 k T}$ is the dimensionless area per defect. Eq. (A4) is a first order, separable ODE which can be easily solved to find $\bar{t}$ as a function of $\bar{z}$ :

$$
\bar{t}=\int_{0}^{\bar{z}} \frac{d \bar{z}^{\prime}}{\sqrt{\bar{z}^{\prime}\left(2-\bar{z}^{\prime}\right)} \exp \left[a\left(\bar{z}_{E}-\bar{z}^{\prime}\right)\right]}
$$

where the range of $\bar{z}$ is $0 \leq \bar{z} \leq \bar{z}_{E}$.

For the sulphate terminated polystyrene spheres at an oil/water interface studied by Kaz et al. [11], $\theta_{E} \approx 110^{\circ}$, $A \approx 5 \mathrm{~nm}^{2}, \gamma=37 \mathrm{mN} \cdot \mathrm{m}^{-1}$ and therefore $a \approx 23$. In Figure 7 a, we show the linear-log plot of $\bar{z}$ vs. $\bar{t}$ calculated from Eq. (A5) for these parameters. We see that the relatively large value of $A$ in this case leads to an essentially logarithmic time dependence for $\bar{z}$ (or $h$ ). On the other hand, for the sulphate or amidine terminated polystyrene spheres at an air/water interface studied by Boniello et al. [17], $\theta_{E} \approx 45^{\circ}, A \approx 0.18 \mathrm{~nm}^{2}, \gamma=71 \mathrm{mN} \cdot \mathrm{m}^{-1}$ and therefore $a \approx 1.6$. In Figure $7 \mathrm{~b}$, we show the linear-log plot of $\bar{z}$ vs. $\bar{t}$ calculated from Eq. (A5) for these parameters. We see that the relatively small value of $A$ in this case leads to a non-logarithmic time dependence for $\bar{z}$ (or $h)$.
[1] R. Aveyard, B. P. Binks, and J. H. Clint, Emulsions stabilised solely by colloidal particles, Curr. Opin. Colloid Interf. Sci. 100, 503 (2003).

[2] A. D. Dinsmore, M. F. Hsu, M. G. Nikolaides, M. Marquez, A. R. Bausch, and D. A. Weitz, Colloidosomes: selectively permeable capsules composed of colloidal particles, Science 298, 1006 (2002).

[3] E. Dickinson, Food emulsion and foams: stabilization by particles, Curr. Opin. Colloid Interf. Sci. 15, 40 (2010).

[4] K. P. Velikov and O. D. Velev, in Colloidal Particles at Liquid Interfaces, edited by B. P. Binks, , and T. S. Horozov (Cambridge Univ. Press, Cambridge, 2006) Chap. 7, p. 225,1 st ed.

[5] J. Forth, P. Y. Kim, G. Xie, X. Liu, B. A. Helms, and T. P. Russell, Building reconfigurable devices using complex liquid-fluid interfaces, Adv. Mater. 31, 1806370 (2019).

[6] P. Aussillous and D. Quere, Liquid marbles, Nature 411, 924 (2001).

[7] K. Stratford, R. Adhikari, I. Pagonabarraga, J.-C. Desplat, and M. E. Cates, Colloidal jamming at interfaces: a route to fluid-bicontinuous gels, Science 309, 2198 (2005).

[8] E. M. Herzig, K. A. White, A. B. Schofield, W. C. K.
Poon, and P. S. Clegg, Bicontinuous emulsions stabilized solely by colloidal particles, Nat. Mater. 6, 966 (2007).

[9] B. P. Binks and R. Murakami, Phase inversion of particlestabilized materials from foams to dry water, Nat. Mater. 5, 865 (2006).

[10] P. Pieranski, Two-dimensional interfacial colloidal crystals, Phys. Rev. Lett. 45, 569 (1980).

[11] D. M. Kaz, R. McGorty, M. Mani, M. P. Brenner, and V. N. Manoharan, Physical ageing of the contact line on colloidal particles at liquid interfaces, Nat. Mater. 11, 138 (2012).

[12] C. Huh and L. E. Scriven, Hydrodynamic model of steady movement of a solid/liquid/fluid contact line, J. Colloid Interface Sci. 35, 85 (1971).

[13] P.-G. deGennes, Wetting: statics and dynamics, Rev. Mod. Phys. 57, 827 (1985).

[14] J. A. Marsh, S. Garoff, and E. B. Dussan V, Dynamic contact angles and hydrodynamics near a moving contact line, Phys. Rev. Lett. 70, 2778 (1993).

[15] T. D. Blake and J. M. Haynes, Kinetics of liquid/liquid displacement, J. Colloid Interface Sci. 30, 421 (1969).

[16] T. D. Blake, The physics of moving wetting lines, J. Colloid Interface Sci. 299, 1 (2006).

[17] G. Boniello, C. Blanc, D. Fedorenko, M. Medfai, N. B. 
Mbarek, M. In, M. Gross, A. Stocco, and M. Nobili, Brownian diffusion of a partially wetted colloid, Nat. Mater. 14, 908 (2015).

[18] A. Wang, W. B. Rogers, and V. N. Manoharan, Effects of contact-line pinning on the adsorption of nonspherical colloids at liquid interfaces, Phys. Rev. Lett. 119, 108004 (2017).

[19] S. Coertjens, R. De Dier, P. Moldenaers, L. Isa, and J. Vermant, Adsorption of ellipsoidal particles at liquidliquid interfaces, Langmuir 33, 2689 (2017).

[20] H. Lehle, E. Noruzifar, and M. Oettel, Ellipsoidal particles at fluid interfaces, Eur. Phys. J. E 26, 151 (2008).

[21] J. de Graaf, M. Dijkstra, and R. van Roij, Adsorption trajectories and free-energy separatrices for colloidal particles in contact with a liquid-liquid interface, J. Chem. Phys. 132, 164902 (2010).

[22] F. Gunther, S. Frijters, and J. Harting, Timscales of emulsion formation caused by anisotropic particles, Soft Matter 10, 4977 (2014).

[23] J. C. Loudet, A. M. Alsayed, J. Zhang, and A. G. Yodh, Capillary interactions between anisotropic colloidal particles, Physical Review Letters 94, 018301 (2005).

[24] J. C. Loudet, A. G. Yodh, and B. Pouligny, Wetting and contact lines of micrometer-sized ellipsoids, Phys. Rev. Lett. 97, 018304 (2006).

[25] E. P. Lewandowski, M. Cavallaro Jr., L. Botto, J. C. Bernate, V. Garbin, and K. J. Stebe, Orientation and self-assembly of cylindrical particles by anisotropic interactions, Langmuir 26, 15142 (2010).

[26] L. Botto, E. P. Lewandowski, J. M. Cavallaro, , and K. J. Stebe, Capillary interactions between anisotropic particles, Soft Matter 8, 9957 (2012).

[27] F. Gunther, Computer simulations of emulsions stabilized by anisotropic particles, Ph.D. thesis, Eindhoven University of Technology (2017).

[28] B. J. Newton, K. A. Brakke, and D. M. A. Buzza, Influence of magnetic field on the orientation of anisotropic magnetic particles at liquid interfaces, Phys. Chem. Chem. Phys. 16, 26051 (2014).

[29] B. J. Newton and D. M. A. Buzza, Magnetic cylindrical colloids at liquid interfaces exhibit non-volatile switching of their orientation in an external field, Soft Matter 12, 5285 (2016).

[30] K. A. Brakke, The surface evolver, Exp. Math. 1, 141 (1992).

[31] B. J. Newton, R. Mohammed, G. B. Davies, L. Botto, and D. M. A. Buzza, Capillary interaction and selfassembly of tilted magnetic ellipsoidal particles at liquid interfaces, ACS Omega 3, 14962 (2018).

[32] S. Coertjens, P. Moldenaers, J. Vermant, and L. Isa, Contact angles of microellipsoids at fluid interfaces, Langmuir 30, 4289 (2014).

[33] M. Doi and S. F. Edwards, The Theory of Polymer Dynamics (Oxford University Press, 1986).

[34] J. Happel and H. Brenner, Low Reynolds Number Hydrodynamics (Prentice-Hall, 1965).

[35] R. Kubo, The fluctuation-dissipation theorem, Rep. Prog. Phys. 29, 255 (1966).

[36] J. C. Berg, Wettability 1st Ed (CRC Press, 1993).

[37] A. Wang and V. N. Manoharan, private communication.

[38] J. D. Feick and D. Velegol, Measurements of charge nonuniformity on polystyrene latex particles, Langmuir 18, 3454 (2002).

[39] J. D. Feick, N. Chukwumah, A. E. Noel, and D. Velegol, Altering surface charge nonuniformity on individual colloidal particles, Langmuir 20, 3090 (2004). 